\title{
Insufficienza renale acuta del postpartum: una diagnosi complessa?
}

\author{
Giuseppe Gernone, Francesco Papagno, Vito Pepe, Francesco Soleti, Michele Giannattasio \\ Per conto del Gruppo di Studio Rene e Gravidanza della Società Italiana di Nefrologia
}

Struttura Complessa di Nefrologia e Dialisi, ASL BARI, Ospedale Santa Maria degli Angeli, Putignano (BA)

\begin{abstract}
ACUte Renal failure POSTPARTum: a COMPleX diagnosis?
Abstract. The differential diagnosis of postpartum acute renal failure associated with microangiopathic hemolytic anemia and thrombocytopenia includes, among others: severe preeclampsia, eclampsia, HELLP syndrome (hemolysis, elevated liver enzyme, low platelet), acute fatty liver of pregnancy (AFLP), thrombotic thrombocytopenic purpura/hemolytic uremic syndrome associated with pregnancy (TTP/aHUS), acute onset or flare of SLE in pregnancy and catastrophic antiphospholipid syndrome (CAPS). These conditions are potentially life threatening due to the presence of multi-organ dysfunction. The occurrence of an hypercoagulable state with decreasing concentration of ADAMTS 13 in pregnancy and in postpartum increases the risk of developing thrombotic thrombocytopenic purpura (TTP). Yet there is a considerable overlap of the clinical and laboratory tools detecting these conditions, therefore the diagnosis may be problematic even for experienced clinicians. However, it is important to establish an accurate diagnosis as the management and complications of these syndromes may be different. This case highlights the complexity of the differential diagnosis in case of microangiopathic hemolytic anemia and thrombocytopenia associated with pregnancy and the role of plasma exchange in their management.
\end{abstract}

Key words: Pregnancy, Acute Renal Failure, Thrombotic microangiopathy, Plasma exchange

Conflict of interest: None.

Ricevuto: 20 Novembre 2012; Accettato: 5 Febbraio 2013

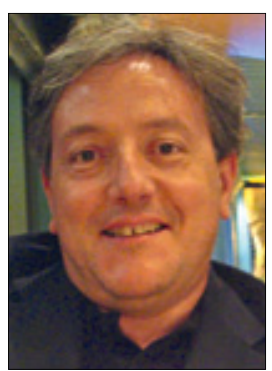

Giuseppe Gernone

\section{Introduzione}

L'insufficienza renale acuta (IRA) del postpartum rientra in una serie di condizioni cliniche, severe, associate ad elevata morbilità e mortalità materno-fetale, che, pur presentando meccanismi ezio-patogenetici differenti, hanno in comune diversi segni clinici e di laboratorio che rendono difficile la diagnosi differenziale.

Tali condizioni condividono una serie di anomalie fisiopatologiche, quali vasocostrizione, attivazione e consumo di piastrine, emolisi intravascolare e trombosi del microcircolo, ridotta perfusione tissutale, responsabili di quadri clinici diversi tra cui la preeclampsia grave, la sindrome HELLP (hemolysis, elevated liver enzyme, low platelet), la acute fatty liver of pregnancy (AFLP), la porpora trombotica trombocitopenia/sindrome emolitico-uremica associata alla gravidanza (TTP/aHUS); l'esordio acuto oflare di LES in gravidanza e la sindrome catastrofica da anticorpi antifosfolipidi (CAPS).

Presentiamo un caso di IRA postpartum caratterizzato da anemia, anomalie della funzione epatica, piastrinopenia in assenza di una storia di ipertensione e proteinuria.

\section{Descrizione}

Paziente (pz) di anni 26, primipara, con gravidanza gemellare monocoriale biamniotica, senza precedenti nefrologici degni di nota e normotesa. Si ricovera alla $37^{\circ}$ settimana in Ostetricia per algie pelviche e riscontro d'iniziale travaglio di parto. Funzione renale ed emocromo sono nella norma; è presente modesto incremento di ALT. Dopo una settimana il travaglio non si arresta e la pz viene sottoposta a taglio cesareo con I e II nato di sesso maschile (peso corporeo rispettivamente di 2,630 g e 2,160 g). L'esame urine del giorno precedente il parto evidenzia: PS 1,010, proteine $25 \mathrm{mg} / \mathrm{dL}$ e sedimento attivo con eritrociti 5-10 p.c.m., leucociti $>40$ p.c.m., discreta batteriuria e pus +++ .

Il giorno successivo al parto la diuresi si contrae; gli esami di funzionalità renale orientano verso un'IRA del postpartum (creatininemia $3.09 \mathrm{mg} / \mathrm{dL}$ ). Vi è anche leucocitosi, grave anemia $(\mathrm{Hgb} 5.8 \mathrm{~g} / \mathrm{dL})$ e piastrinopenia $(56,000 / \mu \mathrm{L})$; sono presenti, inoltre, anomalie della coagulazione ed incremento dei D-Dimeri (Tab. I). L'ecocardiogramma evidenzia versamento pericardico lieve con contrattilità globale del ventricolo sinistro conservata ed atrio sinistro modicamente dilatato. La radiografia del torace mostra versamento pleurico basale bilaterale più evidente a sinistra mentre l'ecografia dell'addome 
TABELLA I - RISULTATI DEI TEST DI LABORATORIO/RRT/DIURESI

\begin{tabular}{|c|c|c|c|c|c|c|c|c|c|c|}
\hline $\begin{array}{l}\text { Test } \\
\text { (valori normali) }\end{array}$ & In Ost. & Out Ost. & In Nefr. & $2^{\circ} \mathrm{g}$ & $3^{\circ} \mathrm{g}$ & $4^{\circ} \mathrm{g}$. & $7^{\circ} \mathrm{g}$. & $8^{\circ} \mathrm{g}$. & $16^{\circ} \mathrm{g}$. & $30^{\circ} \mathrm{g}$. \\
\hline $\begin{array}{l}\text { Emoglobina }(\mathrm{g} / \mathrm{dL}) \\
(13-16.5)\end{array}$ & 11.4 & 5.8 & 8.7 & 8 & 7.2 & 7.9 & 7.1 & 6 & 6.5 & 8.6 \\
\hline $\begin{array}{l}\text { Piastrine }\left(10^{3} / \mu \mathrm{L}\right) \\
(150-400)\end{array}$ & 266 & 56 & 61 & 44 & 40 & 39 & 81 & 141 & 354 & 297 \\
\hline $\begin{array}{l}\text { Leucociti }\left(10^{3} / \mu \mathrm{L}\right) \\
(4-10)\end{array}$ & 8.6 & 31.2 & 27.5 & 20.8 & 13.4 & 10.7 & 12.08 & 14.1 & 6.18 & 5.7 \\
\hline $\begin{array}{l}\text { Azotemia }(\mathrm{mg} / \mathrm{dL}) \\
(<50)\end{array}$ & 23 & 108 & 124 & 157 & 166 & 148 & 94 & 123 & 137 & 77 \\
\hline Creatinina $(\mathrm{mg} / \mathrm{dL})(<1)$ & 0.78 & 3.09 & 4.4 & 5.5 & 6.7 & 6.7 & 6.7 & 8.1 & 10.6 & 3.2 \\
\hline $\begin{array}{l}\text { Glicemia } \mathrm{mg} / \mathrm{dL} \\
(70-100)\end{array}$ & N.D. & 89 & 64 & 64 & 73 & 89 & 95 & N.D. & 93 & N.D. \\
\hline $\begin{array}{l}\text { Uricemia }(\mathrm{mg} / \mathrm{dL}) \\
(<6)\end{array}$ & N.D. & 10.9 & 12.5 & 14.1 & N.D. & 10.6 & N.D. & N.D. & 10.1 & 3.3 \\
\hline $\begin{array}{l}\text { Bilirubina Tot. } \\
(\mathrm{mg} / \mathrm{dL})(<1)\end{array}$ & N.D. & N.D. & 1.87 & 2.03 & 1.64 & 1.62 & 1.15 & N.D. & 0.62 & 0.37 \\
\hline $\begin{array}{l}\text { Bilirubina Dir. } \\
(\mathrm{mg} / \mathrm{dL})(<0.3)\end{array}$ & N.D. & N.D. & 1.37 & 1.55 & 1.07 & 0.87 & 0.46 & N.D. & 0.56 & N.D. \\
\hline $\begin{array}{l}\mathrm{AST} \\
(\mathrm{UI} / \mathrm{L})(<40)\end{array}$ & N.D. & N.D. & 379 & 398 & 170 & 84 & 40 & 33 & 22 & 13 \\
\hline $\begin{array}{l}\text { ALT } \\
(\mathrm{UI} / \mathrm{L})(<65)\end{array}$ & 68 & N.D. & 654 & 603 & 365 & 218 & 71 & 53 & 34 & 27 \\
\hline $\begin{array}{l}\text { LDH (U/L) } \\
(100-190)\end{array}$ & N.D. & N.D. & 2.117 & 2.925 & 2.525 & 1.894 & 777 & 375 & 345 & N.D. \\
\hline $\begin{array}{l}\text { FDP }(\mathrm{mg} / \mathrm{mL}) \\
(<10)\end{array}$ & N.D. & N.D. & 44 & 43 & 35 & 20 & 18 & N.D. & 10 & N.D. \\
\hline $\begin{array}{l}\text { D-Dimeri (ng/mL) } \\
(68-494)\end{array}$ & N.D. & 1451 & N.D. & 9.791 & 7.696 & 5.365 & 2.603 & N.D. & 830 & N.D. \\
\hline Fibrinogeno $(\mathrm{mg} \%)(200-400)$ & N.D. & N.D. & 368 & 350 & 387 & N.D. & 360 & N.D. & N.D. & N.D. \\
\hline PTT (sec.) $(<44)$ & N.D. & N.D. & 41 & 37 & 65 & 45 & 38 & N.D. & N.D. & N.D. \\
\hline $\begin{array}{l}\text { INR } \\
\text { PT (70\%-100\%) }\end{array}$ & N.D. & 1.29 & 95 & 95 & 91 & N.D. & 94 & 93 & N.D. & N.D. \\
\hline $\begin{array}{l}\text { DIC score } \\
\text { (5-8 DIC probabile) }\end{array}$ & / & l & & 5 & 5 & 4 & 3 & l & l & l \\
\hline C3 $(g / L)(0.9-1.8)$ & N.D. & N.D. & 0.68 & 0.73 & N.D. & N.D. & N.D. & N.D. & N.D. & N.D. \\
\hline $\begin{array}{l}\text { Aptoglobina }(\mathrm{g} / \mathrm{L}) \\
(0.3-2)\end{array}$ & N.D. & N.D. & N.D. & 0.29 & N.D. & N.D. & 0.35 & N.D. & 1.04 & N.D. \\
\hline RRT & 1 & / & l & HD & $\mathrm{HD} / \mathrm{PE}$ & $\mathrm{HD} / \mathrm{PE}$ & $\mathrm{HD} / \mathrm{PE}$ & $\mathrm{HD} / \mathrm{PE}$ & HD & 1 \\
\hline Diuresi $\mathrm{mL} / 24$ ore & N.D. & N.D. & N.D. & 200 & 300 & 450 & 600 & 700 & 2.200 & 2.000 \\
\hline
\end{tabular}

N.D., non disponibile; RRT, renal replacement therapy; HD, emodialisi; PE, plasma exchange

evidenzia modesto versamento addominale e "segni di nefropatia" con aumento delle resistenze intraparenchimali. La pz viene sottoposta ad emotrasfusione di globuli rossi concentrati (GRC) (2 U), a terapia diuretica con Furosemide $60 \mathrm{mg}$ due volte al dì ed infonde eparina. Esegue consulenza nefrologica che conclude per IRA oligoanurica con stato anasarcatico e dispone il trasferimento in nefrologia. La pressione arteriosa (PA) è 140/80 mm/Hg.

All'ingresso in nefrologia la pz è vigile, orientata, non è dispnoica, riferisce nausea e cefalea. La PA è $155 / 85 \mathrm{~mm} / \mathrm{Hg}$, non c'è febbre; i parametri di funzionalità renale mostrano ulteriore peggioramento, non è presente inversione degli elettroliti urinari. Il grado di anemizzazione migliora dopo trasfusione ma sono ancora presenti leucocitosi e piastrinopenia; gli FDP sono aumentati mentre il fibrinogeno è normale. Si riscontra un aumento delle transaminasi (AST 379 UI/L, ALT 654 $\mathrm{UI} / \mathrm{L}$ ), della bilirubina (totale: $1.87 \mathrm{mg} \%$; diretta: $1.37 \mathrm{mg} \%$ ), e dell'LDH (2,117 U/L). Vi è una moderata acidosi metabolica ed una modesta ipoglicemia asintomatica $(64 \mathrm{mg} / \mathrm{dL})$. È incrementata la dose di Furosemide (100 mg ev ed altri $100 \mathrm{mg}$ a distanza di 2 ore). Persiste, tuttavia, l'oligoanuria, si pratica copertura antibiotica con cefalosporine e si prosegue la somministrazione di eparina a basso peso molecolare (Enoxaparina 4,000 UI/die).

Il giorno successivo è ulteriormente peggiorata la creatininemia: $5.5 \mathrm{mg} / \mathrm{dL}$; compare febbricola che perdurerà nei giorni 
successivi; la conta piastrine è $44,000 / \mu \mathrm{L}$. Notevole è l'incremento dei D-Dimeri (9,791 ng/mL). Enzimi epatici, LDH e glicemia appaiono invariati, il test di Coombs indiretto è positivo, l'aptoglobina è inferiore alla norma, la PCR è aumentata. È presente ipoC3. L'esame delle urine evidenzia: PS 1,004, proteine $168 \mathrm{mg} / \mathrm{dL}$ e moltissime emazie nel sedimento. Per il persistere dello stato di IRA e dell'anuria, la pz viene sottoposta ad emodialisi.

In III giornata persiste l'oliguria, peggiora il grado di anemizzazione (Hb $7.2 \mathrm{~g} / \mathrm{dL}$ ) e la piastrinopenia si attesta su 40,000/ $\mu \mathrm{L}$. Gli enzimi epatici mostrano, invece, lieve miglioramento, la glicemia si normalizza. Esegue una nuova trasfusione di GRC (altre ne seguiranno nei giorni successivi per un totale di $6 \mathrm{U})$. Perviene l'esame microscopico su sangue periferico che evidenzia: granulociti neutrofili $85 \%$ e presenza di numerosi schistociti con marcata anisopoichilocitosi della serie eritroide. Al trattamento emodialitico segue, pertanto, trattamento di plasma exchange con plasma fresco congelato che viene effettuato nei successivi 6 giorni. Viene somministrato Desametasone alla dose di $10 \mathrm{mg}$ due volte al dì, $5 \mathrm{mg}$ due volte al dì nei giorni successivi, con graduale sospensione sino alla VII giornata, allorché si assiste al miglioramento della piastrinopenia (normalizzatasi in VIII giornata), ed alla ripresa della diuresi $(800 \mathrm{~mL} / \mathrm{die})$ che si attesterà intorno al litro anche nei giorni successivi. Si assiste ad una relativamente rapida normalizzazione di AST e ALT. In X giornata scompaiono gli schistociti dal sangue periferico e si normalizza la leucocitosi neutrofila. FDP e D-Dimeri appaiono migliorati $(15 \mathrm{mg} / \mathrm{mL}$ e $2,123 \mathrm{ng} /$ $\mathrm{mL}$ rispettivamente), ANA ed Ac. Anti-DNA sono negativi. Dalla XI giornata si assiste ad un progressivo incremento della diuresi che consente la sospensione della terapia emodialitica in XVI giornata. Proseguendo il miglioramento clinico-laboratoristico, la paziente viene dimessa, dopo 1 mese di degenza. Il follow-up nefrologico evidenzia dopo $15 \mathrm{gg}$ : clearance creatinina $51 \mathrm{~mL} / \mathrm{m}$ ', proteinuria $2.8 \mathrm{~g} / 24 \mathrm{~h}, \mathrm{Hgb} 10.4 \mathrm{~g} / \mathrm{dL}$, sono nella norma C3, FDP e D-Dimeri. PA $120 / 80 \mathrm{~mm} / \mathrm{Hg}$. Dopo un mese, viene introdotto in terapia Ramipril $2.5 \mathrm{mg} / \mathrm{die}$ per proteinuria persistente pari a $2.2 \mathrm{~g} / \mathrm{die}$. Dopo 6 mesi si riscontrano: clearance creatinina $86 \mathrm{~mL} / \mathrm{m}$, proteinuria $0.5 \mathrm{~g} / 24 \mathrm{~h}$, $\mathrm{Hgb} 13.2$ g/dL, PA 120/80 mm/Hg. Infine dopo 24 mesi: clearance creatinina $105 \mathrm{~mL} / \mathrm{m}$, proteinuria e microalbuminuria assenti, per cui viene sospeso il trattamento con Ramipril e la pz intraprende una nuova gravidanza condotta normalmente a termine.

\section{Discussione}

Quello descritto è un caso le cui caratteristiche cliniche e di laboratorio consentono di propendere per un quadro di IRA in corso di sindrome HELLP e verosimile concomitante TTPaHUS.

La gravidanza multipla presenta un rischio superiore (3-5 volte) di complicanze materne e fetali (pre-eclampsia [PE] eclampsia, AFLP) che compaiono più precocemente (36 settimane) $(1,2)$.

Di recente poi è stato segnalato che il verificarsi di uno stato d'ipercoagulabilità associato ad una concentrazione ridotta di ADAMTS 13, in gravidanza e nel postpartum, aumenta il ri- schio di sviluppare TTP $(3,4)$.

L'IRA in gravidanza, tuttavia, è un evento raro, la sua incidenza si è ridotta da 1/3,000-1/15,000 gravidanze degli anni ' 60 all'attuale $1 / 20,000$ gravidanze. In particolare, PE grave/ HELLP, TTP/aHUS, ed AFLP sono spesso causa di IRA in gravidanza. Prakash riporta che PE grave e sindrome HELLP sono all'origine di circa il $40 \%$ dei casi di IRA in gravidanza (5).

La sindrome HELLP ha un'incidenza di 1-2 casi per 1,000 gravidanze ed una prevalenza del 10\%-20\% nelle donne con PE grave/eclampsia, queste ultime si verificano nel $7.5 \%$ di tutte le gravidanze. Circa il 70\% dei casi di HELLP ha luogo tra 28 e 36 settimane di gravidanza, la restante parte, di solito, entro 48 ore dal parto. TTP-aHUS mostrano un'incidenza minore di 1:100,000 gravidanze (6-7) ed AFLP 1:20,000 (1). TTP-aHUS, PE grave/HELLP ed AFLP sono dunque le patologie principali da prendere in considerazione allorché vi sia IRA associata ad anemia emolitica microangiopatica e trombocitopenia $(8,9)$. La diagnosi differenziale, per quanto complessa, è fondamentale in quanto la TTP-aHUS conduce ad insufficienza renale progressiva e morte. Sicché nel sospetto clinico di TTP-aHUS è richiesto un intervento urgente mediante plasma exchange $(3,10)$, mentre PE, HELLP ed AFLP tipicamente tendono a risolversi spontaneamente dopo il parto. La sovrapposizione dei dati clinici e di laboratorio: trombocitopenia, anemia emolitica microangiopatica, sintomi neurologici ed insufficienza renale, rende spesso la diagnosi differenziale difficile o impossibile. Per di più tali patologie (TTP-aHUS ed HELLP) possono essere concomitanti (11). Nella sindrome HELLP è frequente il riscontro d'insufficienza renale più severa, sebbene nella maggior parte dei casi la diuresi sia conservata e l'insufficienza renale, insieme alle altre manifestazioni, migliori spontaneamente entro 72 ore dal parto. Alcuni rari casi possono complicarsi con IRA oligo-anurica tale da richiedere l'emodialisi (12).

Per la diagnosi di HELLP è necessario che siano presenti (13): Anemia emolitica microangiopatica con presenza di schistociti ed altri segni di emolisi.

Conta piastrinica $\leq 100,000$ cellule $/ \mu \mathrm{L}$

Bilirubina totale $\geq 1.2 \mathrm{mg} / \mathrm{dL}$

$\mathrm{AST} \geq 70 \mathrm{UI} / \mathrm{L}$.

La diagnosi di TTP-aHUS è, invece, generalmente basata sul riscontro di:

anemia emolitica microangiopatica più severa

Trombocitopenia spesso estrema

IRA di vario grado persistente oltre 72 ore dopo il parto

Alterazioni neurologiche

Febbre (14)

PE-HELLP sono assai più frequenti e sono solitamente precedute da ipertensione arteriosa e proteinuria. TTP-aHUS deve essere sospettata in tutte le donne in gravidanza con grave trombocitopenia, anemia grave ed elevati livelli di LDH (che riflette sia l'emolisi sia il danno ischemico tissutale) ma con elevazione modesta di AST (11).

Le anomalie della coagulazione, inoltre, sono differenti. La HELLP è associata a trombocitopenia, e nei casi più gravi, a coagulazione intravascolare disseminata (DIC). Al contrario, la TTP-aHUS è associata a piastrinopenia isolata ed una percentuale più elevata di schistociti $(2 \%-5 \%)$ rispetto alla 
HELLP $(<1 \%)$. Per ultimo il deficit di ADAMTS-13 è raro o assente in corso di HUS o HELLP (11).

Altra difficoltà di diagnostica differenziale sono rappresentate dalla notevole sovrapposizione clinica tra AFLP ed HELLP. L'evidenza di grave insufficienza epatica, l'ipoglicemia, l'encefalopatia ed una coagulopatia spesso associata a DIC, aminotransferasi $<500 \mathrm{UI} / \mathrm{L}$, un notevole incremento della bilirubina totale, la leucocitosi, l'aumento del tempo di protrombina (PT), l'ipofibrinogenemia, sono indicative di AFLP. L'IRA si inserisce nel quadro di una sindrome epatorenale (15). Poiché essa si manifesta nel III trimestre di , gravidanza la diagnosi precoce e l'immediata interruzione della gravidanza sono essenziali per la sopravvivenza materno-fetale (Tab. II).

Nel nostro caso, seppure presenti alcuni degli elementi citati in corso di AFLP (ad esempio la leucocitosi, l'ipoglicemia e la probabile DIC, oltre agli altri elementi comuni), erano assenti l'incremento persistente del PT, l'ipofibrinogenemia e la più grave iperbilirubinemia, inoltre, l'ecografia epatica non mostrava segni specifici di AFLP e l'epoca d'insorgenza non era tipica.

In esso ricorrono invece tutti gli elementi diagnostici propri della HELLP: anemia emolitica microangiopatica con presenza di schistociti, bassi livelli di aptoglobina, grave piastrinopenia, bilirubina totale $>1.2 \mathrm{mg} / \mathrm{dL}$, AST $>70 \mathrm{UI} / \mathrm{L}$, probabile DIC valutata con DIC score; nonchè il sospetto fondato di TTP-aHUS: IRA di grave entità con oligoanuria persistente, febbre, elevati livelli di LDH. Purtroppo l'indisponibilità di un test per ADAMTS 13 nel nostro laboratorio, e la sua non facile disponibilità nei laboratori in generale, ha condizionato la difficoltà di decidere tra HELLP e TTPaHUS, sebbene successivi lavori hanno evidenziato che i livelli di ADAMTS13 possono essere normali in corso di TTP e bassi nella HELLP (16).

Infine, la sintomatologia neurologica sfumata ed il riscontro

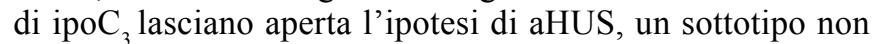
raro in cui la gravidanza (di per sé con decorso normale sotto il profilo materno e fetale) gioca un ruolo scatenante nella genesi del quadro. Essa condivide con le aHUS non correlate a gravidanza una elevata incidenza di deficit di origine genetica od acquisita dei fattori che regolano la via alterna di attivazione del complemento (15). TTP-aHUS sembra dunque la definizione più ragionevole per la maggior parte degli adulti con anemia emolitica microangiopatica e trombocitopenia.

Data l'assenza di miglioramento clinico della pz nel corso delle 72 ore dopo il parto, abbiamo effettuato il trattamento di plasma exchange cui ella ha risposto in modo efficace con risoluzione del quadro di emolisi, la regressione dell'impegno epatico ed il miglioramento del quadro d'insufficienza renale. Il trattamento di plasma exchange va avviato in tutti $\mathrm{i}$ casi di anemia emolitica microangiopatica e trombocitopenia poiché in tali casi è necessario sempre sospettare la diagnosi di TTP-HUS. Il trattamento blocca il consumo delle piastrine responsabili della formazione di trombi e dei sintomi caratteristici della malattia $(17,18)$. Nella TTP vi è carenza, talora causata da autoanticorpi, di una specifica metalloproteasi (ADAMTS 13) devoluta al clivagggio del polimero ultra-

TABELLA II - CONFRONTO DELLE CARATTERISTICHE CLINICHE E DI LABORATORIO TRA TTP / AHUS, SINDROME HELLP ED AFLP

\begin{tabular}{|c|c|c|c|c|}
\hline Manifestazioni cliniche & TTP & aHUS & HELLP & AFLP \\
\hline Anemia emolitica & +++ & +++ & ++ & $+/-$ \\
\hline Piastrinopenia & +++ & +++ & ++ & $+/-$ \\
\hline Anomalie coagulazione -DIC & - & - & $+/-$ & + \\
\hline Insufficienza renale acuta & +++ & +++ & + & ++ \\
\hline Ipertensione & $++/-$ & ++ & +++ & $+/-$ \\
\hline Proteinuria & + (ed ematuria) & ++ & ++ & + \\
\hline Aumento delle transaminasi & $+/-$ & $+/-$ & ++ & +++ \\
\hline Iperbilirubinemia/ittero & ++ & ++ & ++ & +++ \\
\hline Neuropatia & ++ & - & $+/-$ & $+/-$ \\
\hline ADAMTS 13 & ++ & - & - & - \\
\hline LDH & +++ & +++ & + & $+/-$ \\
\hline Febbre & + & + & - & - \\
\hline Effetto del parto & - & - & guarigione & guarigione \\
\hline Trattamento & $\begin{array}{l}\text { Plasma } \\
\text { Exchange }\end{array}$ & $\begin{array}{l}\text { Plasma } \\
\text { Exchange }\end{array}$ & $\begin{array}{l}\text { Ter. Supporto, } \\
\text { Parto }\end{array}$ & $\begin{array}{c}\text { Ter. Supporto, } \\
\text { Parto }\end{array}$ \\
\hline
\end{tabular}


largo del fattore di von Willebrand (ULVWF), nella forma più piccola e meno adesiva del fattore di von Willebrand. Ciò provoca l'accumulo di grandi multimeri del fattore di von Willebrand con conseguente aggregazione piastrinica e deposito di trombi ricchi di piastrine nei tessuti, conseguente danno da stress delle membrane degli eritrociti ed emolisi intravascolare (6). Il plasma exchange interrompe questi processi, poiché rimuove dal plasma gli autoanticorpi anti-ADAMTS13, quando presenti, nonchè i multimeri del fattore di von Willebrand (ULVWF). La sostituzione del plasma del paziente con infusione di plasma normale, inoltre, fornisce la proteasi mancante (ADAMTS13). Sedute quotidiane di plasma exchange devono essere effettuate sino alla normalizzazione della conta piastrinica ed alla cessazione dell'emolisi, (monitorizzando la concentrazione ematica di LDH) $(19,20)$. In media, sono necessarie 7-16 sedute per indurre la remissione, ma vi è grande variabilità in letteratura $(16,21)$. Nel nostro caso sono state sufficienti 7 sedute di plasma exchange.

Martin consiglia l'uso di desametasone in pazienti con HELLP e conta piastrinica inferiore a $100,000 / \mu L$ (22). Recenti evidenze tuttavia suggeriscono di non utilizzare desametasone poichè esso non accelera la risoluzione delle anomalie di laboratorio né riduce il rischio di complicanze materne (23).

Alcuni dati della letteratura segnalano un alto rischio di recidiva di TTP-aHUS in caso di successiva gravidanza, in realtà tale rischio è fondato ma minimo (24), come nel caso descritto.

\section{Conclusioni}

La distinzione tra PE-eclampsia/HELLP, TTP-HUS, AFLP non è sempre possibile a causa dei segni clinici ed esami di laboratorio in comune. In tutti i casi di anemia emolitica microangiopatica e trombocitopenia è necessario sospettare la diagnosi di TTP-HUS ed avviare il trattamento di plasma exchange, che spesso è risolutivo.

\section{Riassunto}

La diagnosi differenziale nei casi d'insufficienza renale acuta del postpartum associata ad anemia emolitica microangiopatica e trombocitopenia, include, tra le altre: preclampsia grave/eclampsia, grave eclampsia, la sindrome HELLP (Hemolysis, Elevated Liver enzyme, Low Platelet), la acute fatty liver of pregnancy (AFLP), la porpora trombotica trombocitopenica/sindrome emolitico-uremica associata alla gravidanza (TTP/aHUS), esordio acuto o flare di LES in gravidanza e la sindrome catastrofica da anticorpi antifosfolipidi (CAPS). Si tratta di condizioni potenzialmente pericolose per la vita data la presenza di disfunzione multiorgano. Il verificarsi di uno stato di ipercoagulabilità e la concentrazione decrescente di ADAMTS 13 in gravidanza e nel post-parto aumentano il rischio di sviluppare porpora trombotica trombocitopenica (TTP). Vi è però una notevole sovrapposizione riguardo la clinica ed i test di laboratorio tra queste condizioni, e quindi la diagnosi può essere un problema anche per clinici esperti. Tuttavia è importante stabilire un'accurata diagnosi poiché la gestione e le complicanze di tali sindromi possono essere differenti. Il caso presentato sottolinea la complessità connessa alla diagnosi differenziale dei quadri clinici che includono anemia emolitica microangiopatica e trombocitopenia connessi alla gravidanza ed il ruolo del plasma exchange nella loro gestione.

Parole chiave: Gravidanza, Insufficienza renale acuta, Microangiopatia trombotica, Plasma-exchange

Dichiarazione di conflitto di interessi: Gli Autori dichiarano di non avere conflitto di interessi.

\author{
Indirizzo degli autori: \\ Dr. Giuseppe Gernone \\ Struttura Complessa di Nefrologia e Dialisi ASL BARI \\ Ospedale Santa Maria degli Angeli \\ Via Cappuccini 7 \\ 70017 Putignano (BA) \\ g.ger@libero.it
}

\section{Bibliografia}

1. Knight M, Nelson-Piercy C, Kurinczuk JJ, Spark P, Brocklehurst P, UK Obstetric Surveillance System. A prospective national study of acute fatty liver of pregnancy in the UK. Gut 2008; 57(7): 951.

2. American College of Obstetricians and Gynecologists. Diagnosis and management of preeclampsia and eclampsia. ACOG Practice Bulletin No 22. Obstet Gynecol 2002; 99: 159-67.

3. Myers L. Postpartum plasma exchange in a woman with su- spected thrombotic thrombocytopenic purpura (TTP) vs. hemolysis, elevated liver enzymes, and low platelet syndrome (HELLP): A case study. Nephrol Nurs J 2010; 37: 399-402.

4. Mannucci PM, Canciani MT, Forza I, Lussana F, Lattuada A, Rossi E. Changes in health and disease of the metalloprotease that cleaves von Willebrand factor. Blood 2001; 98(9): 2730.

5. Prakash J. The kidney in pregnancy: A journey of three decades. Indian J Nephrol 2012; 22 (3): 159-67.

6. Sibai BM. Imitators of severe preeclampsia. Obstet Gynecol 2007; 109(4): 956-66. 
7. Sibai BM. HELLP syndrome. Up to date Ottobre 2012. Disponibile su http://www.uptodate.com/contents/hellpsyndrome? source=search_result\&search $=$ hellp\&selectedTitle= 1\%7E73 (Accesso il 09/11/2012)

8. Sibai BM, Ramadan MK. Acute renal failure in pregnancies complicated by hemolysis, elevated liver enzymes, and low platelets. Am J Obstet Gynecol 1993; 168(6 Pt 1): 1682.

9. McMinn JR, George JN. Evaluation of women with clinically suspected thrombotic thrombocytopenic purpura-hemolytic uremic syndrome during pregnancy. J Clin Apher 2001; 16(4): 202.

10. Ganesan C, Maynard SE. Acute kidney injury in pregnancy: the thrombotic microangiopathies. J Nephrol 2011; 24(5): 554-63.

11. Stella CL, Dacus J, Guzman E, et al. The diagnostic dilemma of thrombotic thrombocytopenic purpura/hemolytic uremic syndrome in the obstetric triage and emergency department: lessons from 4 tertiary hospitals. Am J Obstet Gynecol 2009; 200(4): 381 .

12. Abraham KA, Kennelly M, Dorman AM, Walshe JJ. Pathogenesis of acute renal failure associated with the HELLP syndrome: a case report and review of the literature. Eur J Obstet Gynecol Reprod Biol 2003; 108(1): 99-102.

13. Sibai BM, Ramadan MK, Usta I, Salama M, Mercer BM, Friedman SA. Maternal morbidity and mortality in 442 pregnancies with hemolysis, elevated liver enzymes, and low platelets (HELLP syndrome). Am J Obstet Gynecol 1993; 169(4): 1000.

14. McMinn JR, George JN. Evaluation of women with clinically suspected thrombotic thrombocytopenic purpura-hemolytic uremic syndrome during pregnancy. J Clin Apher 2001; 16(4): 202.

15. Gregorini G. Insufficienza Renale Acuta in gravidanza. In Rene e Gravidanza: Suggerimenti di pratica clinica e di applicazione delle Linee Guida. Disponibile su http://www.nephromeet.com/ web/eventi/nephromeet/index.cfm\# (Accesso il 09/11/2012).

16. Lattuada A, Rossi E, Calzarossa C, Candolfi R, Mannucci PM. Mild to moderate reduction of a von Willebrand factor cleaving protease (ADAMTS-13) in pregnant women with HELLP microangiopathic syndrome. Haematologica 2003; 88(9): 1029-34.

17. Rock GA, Shumak KH, Buskard NA, et al. Comparison of plasma exchange with plasma infusion in the treatment of thrombotic thrombocytopenic purpura. Canadian Apheresis Study Group. N Engl J Med 1991; 325(6): 393.

18. Thompson CE, Damon LE, Ries CA, Linker CA. Thrombotic microangiopathies in the 1980s: clinical features, response to treatment, and the impact of the human immunodeficiency virus epidemic. Blood 1992; 80(8): 1890.

19. George JN. How I treat patients with thrombotic thrombocytopenic purpura: 2010. Blood 2010; 116(20): 4060.

20. George JN. Clinical practice. Thrombotic thrombocytopenic purpura. N Engl J Med 2006; 354(18): 1927.
21. Ruggenenti P, Noris M, Remuzzi G. Thrombotic microangiopathy, hemolytic uremic syndrome, and thrombotic thrombocytopenic purpura. Kidney Int 2001; 60(3): 831.

22. Martin JN Jr, Rose CH, Briery CM. Understanding and managing HELLP syndrome: the integral role of aggressive glucocorticoids for mother and child. Am J Obstet Gynecol 2006; 195(4): 914.

23. Woudstra DM, Chandra S, Hofmeyr GJ, Dowswell T. Corticosteroids for HELLP (hemolysis, elevated liver enzymes, low platelets) syndrome in pregnancy. Cochrane Database Syst Rev 2010.

24. Vesely SK, Li X, McMinn JR, Terrell DR, George JN. Pregnancy outcomes after recovery from thrombotic thrombocytopenic purpura-hemolytic uremic syndrome. Transfusion 2004; 44(8): 1149.

\section{TEST DI VERIFICA}

\section{1) Qual è il trattamento di elezione della TTP:}

a) Infusione di plasma fresco

b) Plasma exchange

c) Desametasone $10 \mathrm{mg}$ b.i.d

2) Nella sindrome HELLP e nella AFLP il trattamento di elezione è rappresentato:
a) Dal parto acuta, sino al momento del parto
c) Infusione di plasma
a) Correlato alla presenza di autoanticorpi
b) Congenito
c) Correlato alla gravidanza
d) Tutte le precedenti

b) Dal trattamento emodialitico, in caso di insufficienza renale

\section{3) Il deficit di ADAMTS 13 in corso di TTP può essere:}

Le risposte corrette alle domande sono pubblicate su questo numero del Giornale di Tecniche Nefrologiche \& Dialitiche Vol. 25, no. 1, pag. 52. 